Perfor mance of Ener gy Har vest er Usi ng I r on- Gal I i um Al I oy i n Free Vi br at i on

\begin{tabular}{|l|l|}
\hline 著者 & Ueno Toshi yuki, Yamada Sot oshi \\
\hline $\begin{array}{l}\text { j our nal or } \\
\text { publ i cat i on t i t l e }\end{array}$ & I EEE Tr ansact i ons on Magget i cs \\
\hline vol une & 47 \\
\hline number & 10 \\
\hline page r ange & $2407-2409$ \\
\hline year & 2011- 10-01 \\
\hline URL & ht t p: //hdl . handl e. net /2297/48304 \\
\hline
\end{tabular}




\title{
Performance of Energy Harvester Using Iron-Gallium Alloy in Free Vibration
}

\author{
Toshiyuki Ueno and Sotoshi Yamada \\ Kanazawa University, Kanazawa, Ishikawa 920-1192, Japan
}

\begin{abstract}
We propose a micro energy-harvesting device, using an iron-gallium alloy (Galfenol), capable of generating electrical energy from ambient vibrations. Galfenol is a ductile magnetostrictive material with a high piezomagnetic constant, good machinability, and a large inverse magnetostrictive effect by which magnetization can be varied by mechanical stress. The device consists of two beams of Galfenol combined with iron yokes, coils, and a bias magnet. A bending force applied at the tip of the cantilever yields a flux increase by tensile stress in one beam, and a flux decreases in the other by compression. The time variation of the flux generates a voltage on the wound coils. This energy harvester has advantages over conventional types of device, such as those using piezoelectric materials, with respect to size, and efficiency, and it has high robustness and low electrical impedance. In addition, the structure needs only a low mechanical force to generate electricity. In this paper, the free vibration characteristic to accrue electric energy effectively is examined. From the experimental results, the energy conversion efficiency in the vibration is inverse proportional to the resonant frequency.
\end{abstract}

Index Terms-Energy harvester, free vibration, Galfenol, inverse magnetostrictive effect.

\section{INTRODUCTION}

$\mathbf{E}$ NERGY-HARVESTING technology generating electrical energy from ambient vibration has been in the spotlight recently because of the development of low power consumption sensors and wireless communication system. The technologies are divided roughly into using piezoelectric materials [1], [2] and moving magnet (electromagnetic induction) [3], [4]. However, at present, there are few commercial products being used effectively. The reasons are low power generation, low efficiency, and poor environmental endurance. For example, piezoelectric materials are brittle with poor robustness to bending and tension. They also suffer from high output impedance, which is a result of their capacitive properties, transfers only small amounts of electrical energy to external loads.

To solve these problems, we propose a microenergy harvester using an iron-gallium alloy (Galfenol, $\mathrm{Fe}_{81.6} \mathrm{Ga}_{18.4}$ ) [5]. Galfenol is an iron-based magnetostrictive material with good machinability and high robustness [6], [7]. It also has a high piezomagnetic constant, a high relative permeability, and a high Curie temperature of over $700^{\circ} \mathrm{C}$. The principle is based on the inverse magnetostrictive effect, where the magnetic flux density inside can be varied by a mechanical load [8]. In addition, the device is remarkably small requires small force to generate sufficient electricity. In this paper, the free vibration characteristic to accrue electric energy effectively is examined.

\section{PrinciPle OF Power GENERATION}

Our proposed energy harvester is based on a structure with two parallel beams using Galfenol (magnetically easy axis in the longitudinal direction) with a wound coil as shown in Fig. 1. Galfenol is stress annealed under a compressive stress [9] to provide built-in uniaxial anisotropy so that flux variations occur under tensile as well as compressive stresses. The ends of the

Manuscript received February 21, 2011; accepted April 21, 2011. Date of current version September 23, 2011. Corresponding author: T. Ueno (e-mail: ueno@ec.t.kanazawa-u.ac.jp).

Color versions of one or more of the figures in this paper are available online at http://ieeexplore.ieee.org.

Digital Object Identifier 10.1109/TMAG.2011.2158303

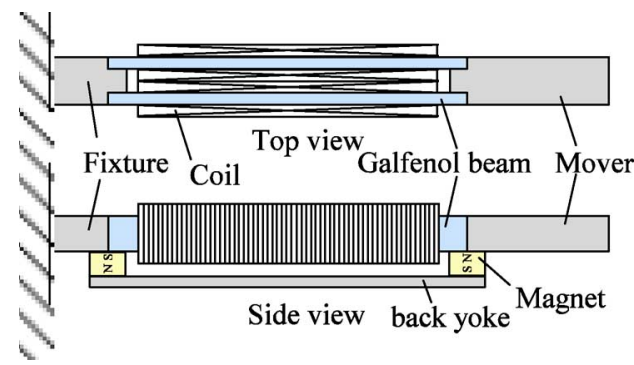

Fig. 1. Configuration of the device.

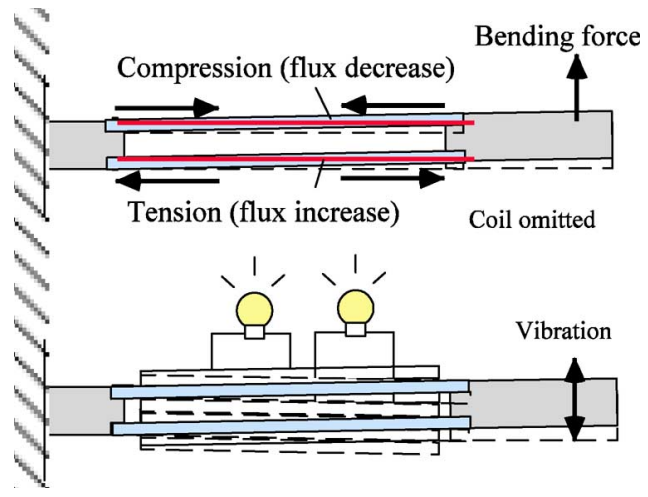

Fig. 2. Principle of energy harvesting (top: forces applied to Galfenol beams by bending force; bottom: electric energy generation by vibration).

two beams are connected to iron yokes by epoxy resin. In addition, one yoke is bonded to a fixture and the other to a mover which oscillates. A permanent magnet with a back yoke is attached to the structure to provide adequate bias flux for the beams. A voltage is generated on the coils as follows. When a bending force is exerted on the mover as shown in the top part of Fig. 2, the structure bends like a cantilever, i.e., a compressive stress is applied to one beam, and a tensile stress is applied to the other in the longitudinal direction. The compression causes a flux decrease and the tension causes a flux increase as a result of the inverse magnetostrictive effect. As shown in the bottom part of Fig. 2, by vibrating the device, the time variations of the fluxes caused by periodic bending deformation generates a voltage on the coils by Faraday's law of induction. 


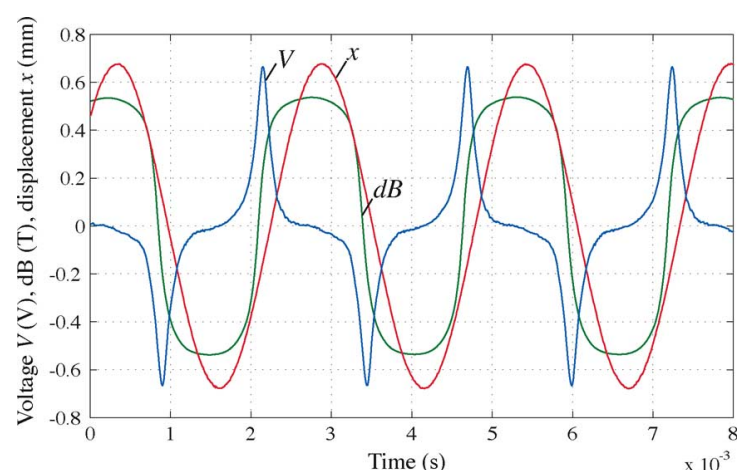

Fig. 3. Time response at forced vibration (frequency $395 \mathrm{~Hz}$ ).
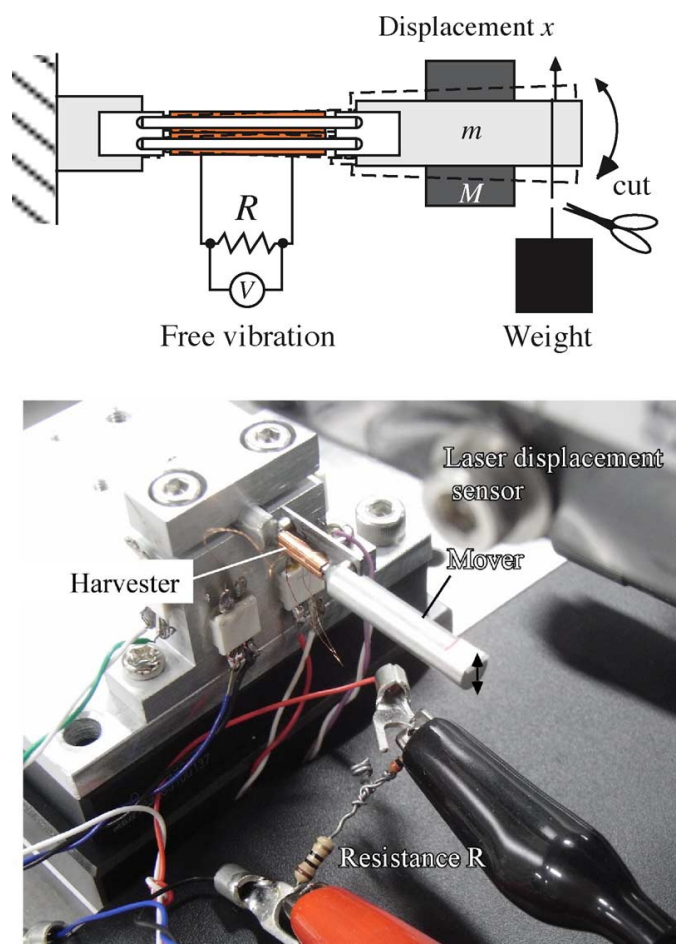

Fig. 4. Experimental setup.

We have verified the principle by a prototype (see picture in Fig. 4). Fig. 3 shows the time responses of displacement $x$ of the mover, generated voltage $V$ on connected resistance of $30 \Omega$, and flux density variation $d B$ in forced vibration (resonance). It is observed that the voltage occurs by bending of the beams. In this case, the flux density inside varies $\pm 0.55 \mathrm{~T}$. The advantages of the harvester are simple configuration and high robustness against external forces because of the ductile properties of Galfenol. In addition, the parallel beams structure requires much smaller force to yield sufficient stress inside the beams compared to the uniaxial force needed in the same direction to yield the same stress. The power generation is high due to high resonance frequency, and the energy conversion efficiency is also high because of the high coupling coefficient of the material.

\section{Free VibRation Characteristics}

The generated voltage is proportional to the frequency due to Faraday's law of induction. Therefore, it is desirable to utilize resonant vibration of high frequency in order to generate high electrical energy efficiently. In fact, the harvester was verified

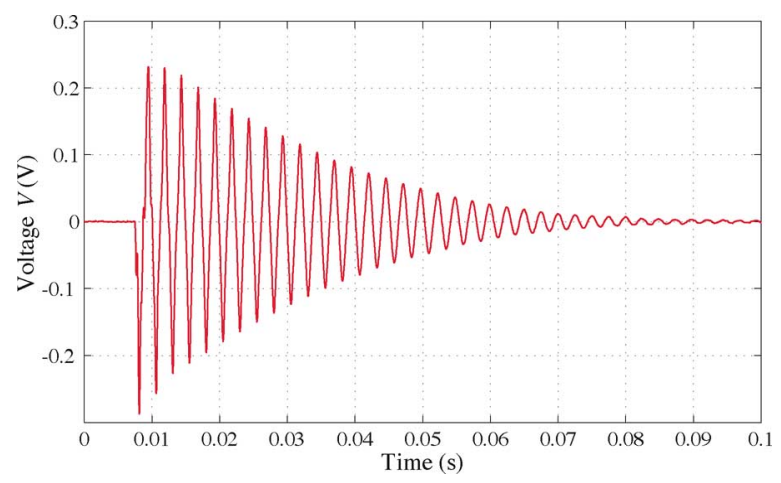

Fig. 5. Generated voltage at free vibration $(f r=392 \mathrm{~Hz})$.

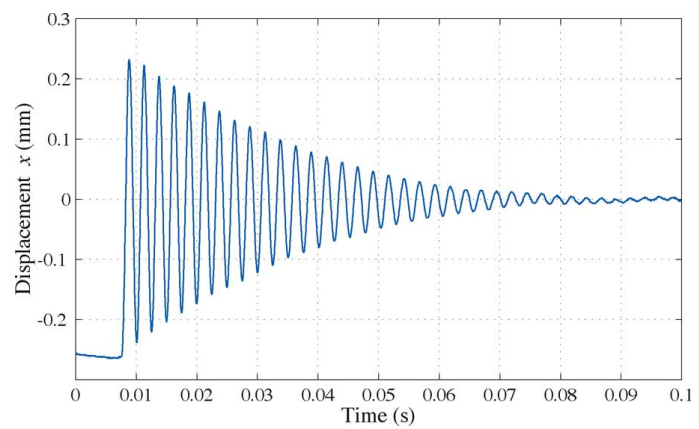

Fig. 6. Displacement at free vibration $(f r=392 \mathrm{~Hz})$.

to provide the maximum output power of $2 \mathrm{~mW}$ at first bending resonance of $395 \mathrm{~Hz}$ at forced vibration as shown in Fig. 3. The harvester also oscillates with resonance in free vibration when mechanical force is exerted on the mover to bend the beams and released suddenly. In practical applications, the free vibration is intermediate to convert low frequency periodic input force of several hertz order to resonance. Here, the output energy and energy conversion efficiency are dependent on the amplitude and resonance in free vibration are examined.

Fig. 4 shows an experimental setup. In the measurement, bending force was exerted on the mover by hanging a weight via string. Then, the free vibration was occurred by cutting the string. The dimension of Galfenol beams are $1.0 \mathrm{~mm} \times 0.5 \mathrm{~mm}$ by $10 \mathrm{~mm}$, and winding coils are 312 turns of $0.05 \mathrm{~mm}$ diameter wire $(15 \Omega)$. The bias magnet is $\mathrm{Nd}-\mathrm{B}-\mathrm{Fe}$ magnet of $2 \mathrm{~mm}$ diameter and $2 \mathrm{~mm}$ length. Two cases of different resonant frequency were compared with and without attaching additional mass $M$ on the mover $m$. The harvester was connected in series to the resistance $R=30 \Omega$ and the generated voltage $V$ was measured by a high-impedance probe $(10 \mathrm{M} \Omega)$. The displacement $x, 2 \mathrm{~mm}$ from the edge of the mover, was measured by a Laser sensor.

Energy conversion efficiency $\eta$ is defined as output electrical energy Wo taken out at the resistance $R$ as Joule loss divided by work $W i$ conducted on the mover as following equation:

$$
\eta=W_{o} / W_{i}=\int_{0}^{t_{f}} \frac{V^{2}}{R} d t / \frac{1}{2} F_{0} X_{0}
$$

where $F_{0}$ and $X_{0}$ are the initial force and displacement to trigger the vibration.

Figs. 5 and 6 show the time responses of $x$ and $V$ without mass, respectively. The maximum voltage $V_{0}$ at beginning is $0.29 \mathrm{~V}$, resonant frequency $\mathrm{fr}$ is $395 \mathrm{~Hz}$ and logarithmic decrement $\Delta$ is 0.081 . Wo is $1.2 \times 10^{-5} \mathrm{~J}$ and $W i$ is $8.9 \times 10^{-5} \mathrm{~J}$; 


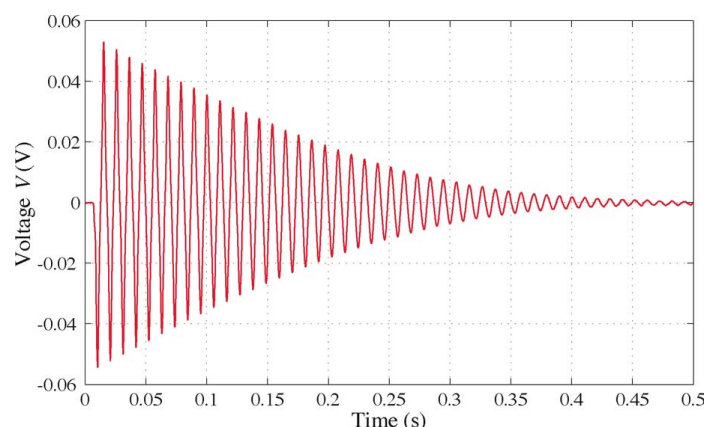

Fig. 7. Generated voltage at free vibration with mass $(f r=94 \mathrm{~Hz})$.

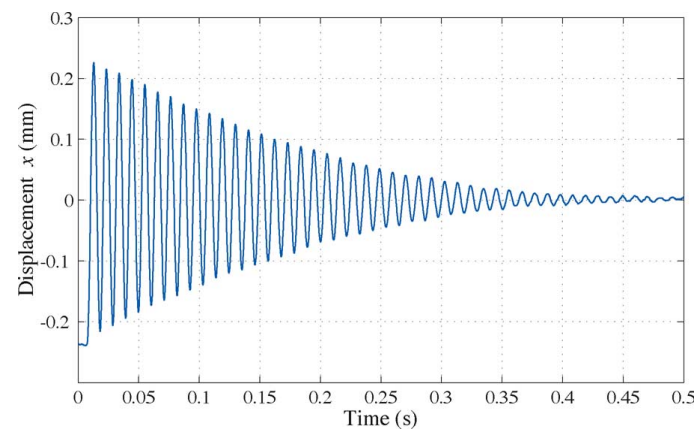

Fig. 8. Displacement at free vibration with mass $(f r=94 \mathrm{~Hz})$.

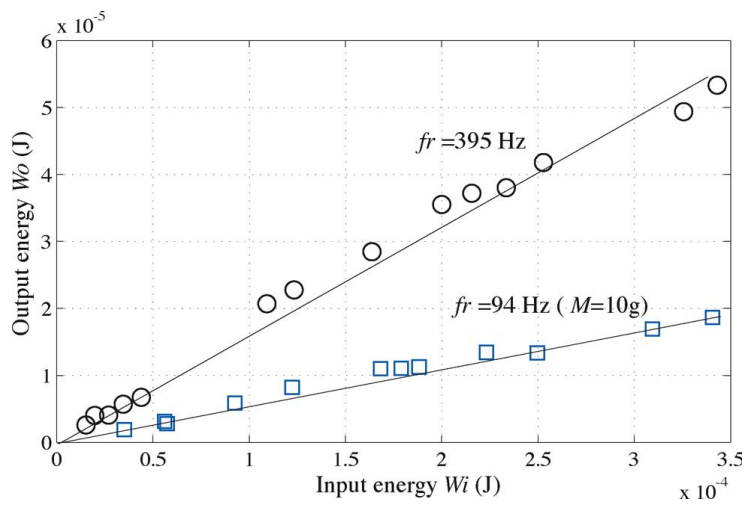

Fig. 9. Comparison of Wi and Wo measured by several initial condition.

thus, $\eta$ is calculated 0.14 . Figs. 7 and 8 show the time responses of $x$ and $V$ with additional $M=10 \mathrm{~g}$, respectively. In this case, $V_{0}$ is reduced to $0.054 \mathrm{~V}$ because of the decrease of $f r$ to $94 \mathrm{~Hz}$, and $\Delta$ is 0.07 . Wo is $0.36 \times 10^{-5} \mathrm{~J}$ and $W i$ is $6.1 \times 10^{-5} \mathrm{~J}$; thus, $\eta$ is reduced to 0.06 . Fig. 9 compares $W o$ and $W i$ with and without mass measured by several initial conditions. $\eta$, the slope of the curve, is regarded constant. This means that $\eta$ does not depend on the amplitude. Average $\eta$ are 0.16 $(16 \%)$ at $f r=395 \mathrm{~Hz}$ and $0.054(5.4 \%)$ at $94 \mathrm{~Hz}$. From the experimental results, the efficiency $\eta$ reduces with the resonant frequency $f r$. The reason is as follows. The voltages $V$ occurs by time variation of flux $\Phi_{m}$ inside the beam proportional to the velocity of the mover:

$$
V=N \frac{d \Phi_{m}}{d t}=d_{H} A_{m} N \frac{d T_{m}}{d t}=K \dot{x} .
$$

Here, $N, T_{m}, A_{m}$, and $d_{H}$ are the numbers of turns of coils, stress, area of beams, and piezomagnetic constants, respectively. Thus, the output energy $W_{o}$ of the joule loss reduces proportional to square of $f r$. On the other hand, the vibration lasts longer inverse proportional to $f r$ as shown in Fig. 6 . Therefore, $\eta$ is considered roughly proportional to $f r$.

The bottom line is that the resonant frequency $f r$ should be higher to accrue electric energy effectively. As shown in Fig. 9, $3.5 \times 10^{-4} \mathrm{~J}$ is obtained by one shot of the vibration. Thus, the harvester can generate the maximum power of $3.5 \mathrm{~mW}$ by ten times vibration per second. For practical applications, conversion mechanism to trigger free vibration by periodical force of low frequency should be developed.

\section{ACKNOWLEDGMENT}

This work was supported by ONR Code 321 MS, the Naval Surface Warfare Center's Magnetic Materials Group (Code 612), and the Murata Science Foundation.

\section{REFERENCES}

[1] S. Cagatay, B. Koc, and K. Uchino, "A $1.6 \mathrm{~mm}$, metal tube ultrasonic motor," IEEE Ultrason., Ferroelectr., Freq. Control, vol. 50, no. 7, pp. 782-786, Jul. 2003.

[2] J. L. Gonzàlez, A. Rubio, and F. Moll, "Human powered piezoelectric batteries to supply power to wearable electronic devices," Int. J. Soc. Mater. Eng. Resour., vol. 10, pp. 33-40, 2002.

[3] T. Buren and G. Troster, "Design and optimization of a linear vibrationdriven electromagnetic micro-power generator," Sen. Actuators A, vol. 135, pp. 765-775, 2007.

[4] C. R. Saha, T. O'Donnell, N. Wang, and P. McCloskey, "Electromagnetic generator for harvesting energy from human motion," Sen. Actuators A, vol. 147, pp. 248-253, 2008.

[5] T. Ueno and S. Yamada, "Micro energy harvester using iron-gallium alloy," in Book Abstract, 55th MMM Conf., 2010.

[6] A. E. Clark, M. Wun-Fogle, and J. B. Restorff, "Magnetostrictive properties of body-centered cubic Fe-Ga and Fe-Ga-Al alloy," IEEE Trans. Magn., vol. 37, no. 5, pp. 3238-3240, Sep. 2000.

[7] R. A. Kellog, A. M. Russell, T. A. Lograsso, A. B. Flatau, A. E. Clark, and M. Wun-Fogle, "Tensile properties of magnetostrictive iron-gallium alloy," Acta Materialia, vol. 52, pp. 5043-5050, 2004.

[8] T. Ueno and T. Higuchi, "Magnetic circuit for stress-based magnetic force control using iron-gallium alloy," IEEE Trans. Magn., vol. 43, no. 6, pp. 2594-2596, Jun. 2007.

[9] M. Wun-Fogle, J. B. Restorff, and A. E. Clark, "Magnetomechanical coupling in stress-annealed Fe-Ga and Fe-Al alloys," IEEE Trans. Magn., vol. 42, no. 10, pp. 3120-3122, Oct. 2006. 